\title{
Analyzing Energy Levels and Gamma Decay Spectrum Phosphorus-32(14d) Isotope Using OXBASH Shell Model Code for Lower HASP Model Space
}

\author{
Email address: \\ phd.shafeghat.h@pnum.ac.ir (H. Shafeghat) \\ ${ }^{*}$ Corresponding author
}

Hamid Shafeghat", Maliheh Teimur, Saeed Mohammadi

Department of Physics, Payame Noor University, Tehran, Iran

To cite this article:

Hamid Shafeghat, Maliheh Teimur, Saeed Mohammadi. Analyzing Energy Levels and Gamma Decay Spectrum Phosphorus-32(14d) Isotope Using OXBASH Shell Model Code for Lower HASP Model Space. Modern Chemistry. Vol. 5, No. 5, 2017, pp. 82-85. doi: 10.11648/j.mc.20170505.12

Received: May 31,2017; Accepted: June 21, 2017; Published: November 5, 2017

\begin{abstract}
In this paper, we have calculated the energy excitation and gamma spectrum for transition strengths $\mathrm{B}(\mathrm{E} 2)$ and $\mathrm{B}(\mathrm{M} 1)$ of the phosphorus-32(14d) isotope in the lower HASP-shell region for the nuclei Phosphorus-32(14d). We have performed this calculation with the isovector $\mathrm{T}=1$ and positive parity states for this isotope, using the shell model code OXBASH for Windows by employing the effective interactions HASP, HASN, VPNP and VPTH. The level schemes and $\mathrm{B}(\mathrm{E} 2)$ and $\mathrm{B}(\mathrm{M} 1)$ values are more reasonable in comparison with the available experimental data.
\end{abstract}

Keywords: Energy Levels, HASP Model Space, OXBASH Code, P Isotopes, Transition Strengths

\section{Introduction}

OXBASH is a powerful computer code for estimating the light and average nuclei energy levels. Using this code, one can compute the energy levels of hundreds of isotopes, and validate the results by comparing them with those obtained by experiments. In addition, studying the energy levels by means of shell model was always a challenge for nuclear physicists, and up to now, different models were proposed for investigating this issue. In this regard, too many computational codes were developed, and OXBASH is one of the recent advanced codes in this area. OXBASH is set of codes for carrying out shell-model calculations with dimensions up to about 100,000 in the J-T scheme and about $2,000,000$ in the M-scheme. OXBASH comes with a library of model spaces and interactions. The OXBASH version 2005-08 for PC was used, which can be installed and used in any operating system.

After selecting the appropriate model based on the valence nucleons, OXBASH lists the possible initial states, and then it develops the linear combination of initial states, by which the desired values for $\mathrm{J}$ and $\mathrm{T}$ are obtained. The number of linear combinations determines the size of $\mathrm{J}-\mathrm{T}$ matrix.
Finally, by selecting desired interaction, the Hamiltonian is formed, and then the calculations are performed.

In this work, because of the quite importance of the Gamma decay spectrum of nuclei ${ }_{15}^{32} P$ in radiation medical, such as studying treatment of polycythemia Vera (excess red blood cells), we try to calculations energy excitation and transition probability in the lower HASP-shell region for the nuclei ${ }_{15}^{32} P$, to test the ability of the present effective interactions in reproducing the experiment in this mass region.

\section{Model Space}

The "model space" indicates the orbitals and truncation within that set of orbitals which is assumed for a given calculation. Generally, the best and most complete results are obtained when the model space is as large as possible. However, the computation time increase exponentially with the size the model space, and empirical hamiltonians are better determined in the smaller model space. Thus the choice of model space is a compromise between what one would like to describe and what is computationally practical [1]. 
As a result, due to existing single proton in $1 \mathrm{~d} 3 / 2$ state for protons as well as three neutrons in this state, we have considered HASP model space which consisted of $1 \mathrm{~d} 3$ / $2,2 s 1 / 2,2 p 3 / 2,1 f 7 / 2$ states.

\section{Hamiltonianism}

The microscopic calculations discussed on two different types of calculations. The first is the mean-field approximation which is applicable to all nuclei but which is based on the restriction to a single Slater determinant. The second is shell-model configuration mixing in which a matrix based upon all possible Slater determinants with in a relatively small subset of valence orbits are diagonalized [1].

Here, we have studied, the first subject which perform explicit calculations of the single- particle energies and density distributions.

\section{Theory and Calculations}

The structure of phosphorus-32(14d) isotope, has been studied in the framework of the shell model using the OXBASH code [1].

For the calculations, the shell model Hamiltonian can be written as [16]

$$
H=\sum_{i} \varepsilon_{i} a_{i}^{\dagger}+\sum_{i} V_{i j k l} a_{i}^{\dagger} a_{j}^{\dagger} a_{i} a_{j}
$$

Where $\varepsilon_{i}$ : is the single-particle energies (SPE) that can be obtained from neighbors of closed shell nuclei having mass $\mathrm{A}=$ closed core +1 .

$V_{i j k l}$ : is the Two-Body Matrix Element (TBME) coupled to good spin and isospin.

$a_{i}^{\dagger} a_{j}^{\dagger}$ :Creation operators (create pair of fermions).

$a_{i} a_{j}$ : Annihilation operators (annihilate pair of fermions).

The TBME where calculated by using Four effective interactions potential HASP [1], HASN [1], VPNP [1] and
VPTH [1].

The reduced transition probability for electric multiple radiation is given by [17]

$$
\begin{aligned}
& B\left(E l, J_{i} \rightarrow J_{f}\right)= \\
& \frac{2 J_{i}+1}{2 J_{f}+1}\left|d^{3} r<f\left\|\hat{\rho}(r) r^{l} Y_{l}(\Omega)\right\| f>\right|^{2}
\end{aligned}
$$

Where $\hat{\rho}(r)$ : is the charge density operator.

$Y_{l}(\Omega)$ : is the spherical harmonics

\section{Excitation Energy}

As reminded of in the earlier section, the principal idea for studying this nuclei lies in the Appropriate of this isotope in the radiation medical science due to the importance of this applications in investigation treatment of polycythemia Vera [15].

First procedure to analyzing the excitation and gamma spectrum of ${ }_{15}^{32} \mathrm{P}$ isotope relies on basic information for running OXBASH code (such as atomic number, neutron number, mass number of desired isotope, isospin, parity, number of valence particles, and the number of levels, in which nucleons can be excited to, are obtained by reference [1]. Four effective interactions potential HASP [1], HASN [1] VPNP [1] and VPTH [1] were employ with HASP model space for the calculations of level excitation energy and transition probabilities.

We have to mention here that ${ }_{15}^{32} P$ has isovector part $\mathrm{T}=1$ and its total angular momentum changes from 0 to 4 .

Figure 1 show the comparison of the experimental excitation energies of ${ }_{15}^{32} P$ with calculated values from HASP, HASN, VPNP and VPTH effective interactions. The effective interactions VPTH gives close results in comparison with the experimental values up to $J^{\pi}=3^{+}$. From Figure 1 we can notice that VPTH are in excellent agreement with the experiment better than HASP, HASN and VPNP.

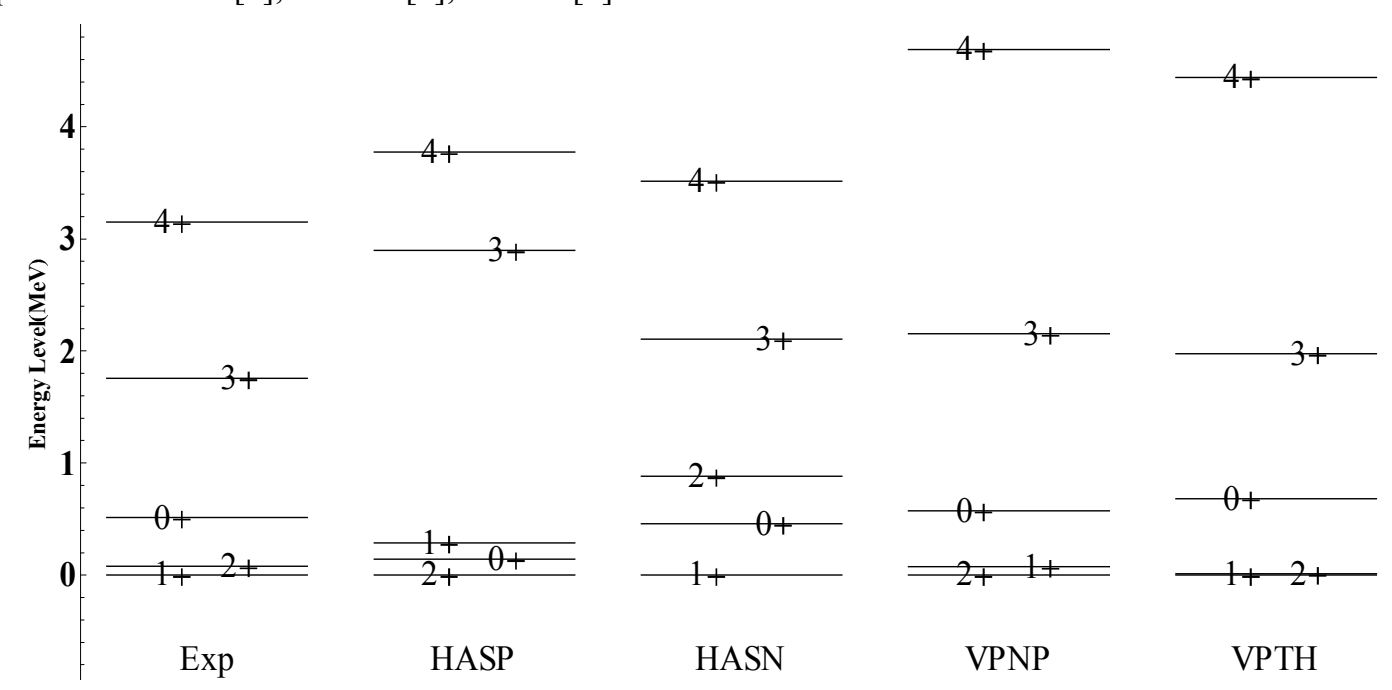

Figure 1. Comparison of the experimental excitation energies taken from Ref. [13] with the present theoretical work using HASP, HASN, VPNP, VPTH effective interactions. 


\section{Interaction Types}

The starting point for shell-model Hamiltonians is the renormalized $\mathrm{G}$ matrix based upon modern $\mathrm{N}-\mathrm{N}$ interactions [8]. When the G matrix is used to calculate the spectra for the HASP-shell nuclei with more than two particles or holes, the agreement with the experimental energy spectra deteriorates rapidly as the number of particles or holes is increased [6]. There were several attempts to find improved empirical Hamiltonians. By 1979 Hasper [14], [8] had obtained empirical Hamiltonians for the lower-part of the HASP-shell. In OXBASH for HASP space model have been proposed 4 different interactions were included $[1,2]$. The calculations were done for all these potentials for ${ }_{15}^{32} P$.

\section{Transition Probability}

HASP model space contains valence orbitals of $1 \mathrm{~d} 3 /$
$2,2 s 1 / 2,2 p 3 / 2,1 f 7 / 2$. Since the transition rates represent a sensitive test for the most modern effective interactions that have been developed to describe HASP-shell nuclei. The transition strengths calculated in this work performed using for each in-band transition by assuming pure E2 and M1 transition. Our results from shell model code and the previous theoretical results using different models are listed in Table 1 for Some of Interaction effective. The same comparison was made, but the result of experimental data is not available, therefore we cannot judge which effective interaction reproduce the experimental data better. Here, we have supposed the effective charges for both of proton and neutron are same and taken to be $0.5 \mathrm{e}$ for the calculations of the transition strengths of ${ }_{15}^{32} P$. Our theoretical results are in excellent agreement with the experimental values for the transitions

$2^{+} \rightarrow 1^{+}$and $3^{+} \rightarrow 1^{+}$using VPTH effective interaction has been summarized in Table 1 .

Table 1. The B(E2) and B(M1) values in the ground-state band of ${ }_{15}^{32} P$. Their units are $e^{2} f m .{ }^{4}$. Exp [13].

\begin{tabular}{|c|c|c|c|c|c|}
\hline \multirow[b]{2}{*}{$J_{i}^{\pi} \rightarrow J_{f}^{\pi}$} & \multicolumn{2}{|l|}{ Exp. } & \multirow{2}{*}{$\begin{array}{l}\text { Present } \\
\text { HASP }\end{array}$} & \multicolumn{2}{|l|}{ Work } \\
\hline & $\begin{array}{l}\text { B(M1) } \\
\text { B(E2) }\end{array}$ & VPTH & & HASN & VPNP \\
\hline $2^{+} \rightarrow 1^{+}$ & $\begin{array}{l}1.613 \\
----\end{array}$ & $0.15 \mathrm{E}+01(\mathrm{M} 1) \quad 0.13 \mathrm{E}+00(\mathrm{E} 2)$ & - & $0.01 \mathrm{E}+00(\mathrm{M} 1) 0.88 \mathrm{E}+00(\mathrm{E} 2)$ & --------- \\
\hline $0^{+} \rightarrow 1^{+}$ & $\begin{array}{l}0.853 \\
-----\end{array}$ & $0.12 \mathrm{E}+01(\mathrm{M} 1) \quad 0.00 \mathrm{E}+00(\mathrm{E} 2)$ & -------- & $0.20 \mathrm{E}+01(\mathrm{M} 1) 0.00 \mathrm{E}+00(\mathrm{E} 2)$ & $0.53 \mathrm{E}+02(\mathrm{M} 1) 0.00 \mathrm{E}+00(\mathrm{E} 2)$ \\
\hline $3^{+} \rightarrow 2^{+}$ & $\begin{array}{l}0.121 \\
38.80\end{array}$ & $0.00 \mathrm{E}+00(\mathrm{M} 1) \quad 0.28 \mathrm{E}+02(\mathrm{E} 2)$ & $0.00 \mathrm{E}+00(\mathrm{M} 1) 0.30 \mathrm{E}+02(\mathrm{E} 2)$ & $0.00 \mathrm{E}+00(\mathrm{M} 1) \quad 0.51 \mathrm{E}+02(\mathrm{E} 2)$ & $0.00 \mathrm{E}+00(\mathrm{M} 1) \quad 0.30 \mathrm{E}+02(\mathrm{E} 2)$ \\
\hline $3^{+} \rightarrow 1^{+}$ & $\begin{array}{l}0.062 \\
58.20\end{array}$ & $0.01 \mathrm{E}+00(\mathrm{M} 1) 0.50 \mathrm{E}+02(\mathrm{E} 2)$ & $0.11 \mathrm{E}+00(\mathrm{M} 1) 0.45 \mathrm{E}+02(\mathrm{E} 2)$ & $0.10 \mathrm{E}+00(\mathrm{M} 1) 0.35 \mathrm{E}+02(\mathrm{E} 2)$ & $0.08 \mathrm{E}+01(\mathrm{M} 1) 0.52 \mathrm{E}+03(\mathrm{E} 2)$ \\
\hline $4^{+} \rightarrow 3^{+}$ & $\begin{array}{l}0.133 \\
7.760\end{array}$ & $0.10 \mathrm{E}+01(\mathrm{M} 1) 0.74 \mathrm{E}+02(\mathrm{E} 2)$ & $0.19 \mathrm{E}+01(\mathrm{M} 1) 0.43 \mathrm{E}+02(\mathrm{E} 2)$ & $0.25 \mathrm{E}+00(\mathrm{M} 1) 0.14 \mathrm{E}+04(\mathrm{E} 2)$ & $0.15 \mathrm{E}+00(\mathrm{M} 1) \quad 0.67 \mathrm{E}+01(\mathrm{E} 2)$ \\
\hline $4^{+} \rightarrow 2^{+}$ & 76.63 & $0.00 \mathrm{E}+00(\mathrm{M} 1) 0.71 \mathrm{E}+02(\mathrm{E} 2)$ & $0.00 \mathrm{E}+00(\mathrm{M} 1) 0.98 \mathrm{E}+02(\mathrm{E} 2)$ & $0.00 \mathrm{E}+00(\mathrm{M} 1) \quad 0.41 \mathrm{E}+02(\mathrm{E} 2)$ & $0.00 \mathrm{E}+00(\mathrm{M} 1) \quad 0.56 \mathrm{E}+02(\mathrm{E} 2)$ \\
\hline $4^{+} \rightarrow 1^{+}$ & $\mathrm{M} 1+\mathrm{E} 2$ & --------- & --------- & --------- & --------- \\
\hline
\end{tabular}

\section{Conclusion}

Full HASP-space shell model calculations were performed using the OXBASH shell model code for Windows. The HASP model space were employed with the effective interactions HASP, HASN, VPNP and VPTH to reproduce the level spectra and transition strengths $\mathrm{B}(\mathrm{E} 2)$ and $\mathrm{B}(\mathrm{M} 1)$ for the phosphorus-32 (14d) Isotope. Excellent agreement was obtained by comparing these calculations with the recently available experimental data for the level spectra using VPTH effective interaction. Calculation of the transition strengths prove that VPTH effective potential is more consistent in reproducing the experiment respect to other interaction potentials for the lower HASP-shell region.

\section{References}

[1] Oxbash for Windows, B. A. Brown, A. Etchegoyen, N. S. Godwin, W. D. M. Rae, W. A. Richter, W. E. Ormand, E. K. Warburton, J. S. Winfield, L. Zhao and C. H. Zimmerman, MSU-NSCL report number 1289.
[2] The Nuclear Shell Model Towards the Drip Lines, B. A. Brown, Progress in Particle and Nuclear Physics 47, 517 (2001).

[3] K.-H Speidel et al Phys Lett B 6591001 (2008).

[4] K.-H Speidel et al Phys Rev C 78, 017304 (2008).

[5] B. H. Wildenthal, E. C. Halbert, J. B. McGrory and T. T. S. Kuo, Phys. Rev. C 4, 1266 (1971).

[6] B. H. Wildenthal, Prog. Part. Nucl. Phys. 11, 5 (1984).

[7] A. E. Stuchnery et al Phys Rev C 74, 054307 (2006).

[8] HASPER, PRC19, 1482 (1979) (1977).

[9] M. Hjorth-Jensen, T. T. S. Kuo and E. Osnes, Phys. Rep. 261, 125 (1995).

[10] T. T. S. Kuo and G. E. Brown, Nucl. Phys. 85, 40 (1966).

[11] T. T. S. Kuo, Nucl. Phys. A103, 71 (1967).

[12] van-der-Poel Nucl. Phys. A373, 81 (82).

[13] C. OUELLET and B. SINGH, Nuclear Data Sheets 112, 2199 (2011).

[14] van-der-Poel Thesis, p. 50 (JAB). 
[15] https://www.radiochemistry.org/nuclearmedicine/radioisotopes /ex_iso_medicine.htm

[16] M. Honma,, B. A. Brown, T. Mizusaki and T. Otsuka, Nucl. Phys. A704, (2002), 134c.

[17] W. Greiner and J. A. Maruhn, "Nuclear Models", SpringerVerlag, Berlin Heidelberg, (1996), printed in Germany.

[18] Asadi, S., Mohammadi, S. and Shafeghat. H, "Analysis of disciplinary of excitation level, Fluorine -19 isotope using OXBASH code," The 8th International Conference on Physical Science PNU University, May. 10-11, 2017/ Shiraz, I. R. Iran.
[19] Abbasnia A., Shafeghat H., Mohammadi S. Calculation of energy level for Calcium isotopes by using of OXBASH code, $23^{\text {rd }}$ Iranian Nuclear Conference, 22-23 Feb 2017 / Islamic Azad University, I. R. Iran.

[20] Shafeghat. H, Sajjadi. Zahra Sadat, Mohammadi, Saeed. Analysis of USD (Universal SD- Shell Interaction) Interaction on level energy of Chlorine-17 and Phosphorous-16 Mirror nucleus using OXBASH code and comparison them with experimental data, The 8th International Conference on Physical Science PNU University, May. 10-11, 2017/ Shiraz, I. R. Iran. 